\section{Windaus, Adolf Otto Reinhold}

\section{W. Hubl}

Ehem. Krankenhaus Dresden-Friedrichstadt, Institut für Klinische Chemie und Labormedizin, Dresden, Deutschland

Lebensdaten Deutscher Mediziner, geboren am 25. Dezember 1876 in Berlin, gestorben am 9. Juni 1959 in Göttingen. Windaus besuchte das Französische Gymnasium in Berlin. Ab 1895 studierte er Medizin an den Universitäten in Berlin und Freiburg im Breisgau. Nach dem Physikum im Jahr 1897 wandte er sich der Chemie zu und promovierte im Jahr 1899 an der philosophischen Fakultät Freiburg über „Neue Beiträge zur Kenntnis der Digitalisstoffe“. Nach zwei Jahren als Mitarbeiter von Emil Fischer (1852-1919) in Berlin kehrte er im Jahr 1901 nach Freiburg zurück und habilitierte zwei Jahre später über > Cholesterin. Von 1906-1913 war er Professor in Freiburg und ging danach bis 1915 an das Institut für Angewandte Medizinische Chemie an der Universität Innsbruck. Von 1915 bis zu seiner Emeritierung im Jahre 1944 hatte er den Lehrstuhl für Chemie der Universität Göttingen inne.

Verdienste Windaus war einer der führenden Naturstoffchemiker seiner Zeit. Besonderes Interesse fanden hierbei seine
Forschungstätigkeiten über wesentliche Grundlagen der Steroide und des Zusammenhanges mit $\triangleright$ Gallensäuren und Saponinen. Er klärte die chemische Struktur verschiedener Steroide, wie $>$ Cholesterin und Ergosterin, auf. Darüber hinaus gelang es ihm, die Struktur verschiedener Vitamine des B-Komplexes und der D-Gruppe (Vitamin D2 und D3; „Vigantol") aufzuklären und diese zu synthetisieren. Im Jahr 1928 erhielt Adolf Windaus den Nobelpreis für Chemie für seine Verdienste um die Erforschung des Aufbaus der Sterine und ihres Zusammenhangs mit den Vitaminen. Windaus war Mitglied der Akademie der Wissenschaften zu Göttingen, erhielt zahlreiche Ehrendoktortitel und Auszeichnungen, wie den Orden Pour le Merite, die Louis-Pasteur-Medaille und die Goethe-Medaille.

\section{Literatur}

Dimroth K (1976) Das Portrait: Adolf Windaus 1876-1959. Chemie in unserer Zeit. 10 S 175-179

Nobel Lectures, Chemistry 1922-1941, Elsevier Publishing Company, Amsterdam, 1966 Haas J (2006) Vigantol. Adolf Windaus und die Geschichte des Vitamin D. Heidelberger Schriften zur Pharmazieund Naturwissenschaftsgeschichte, Bd 20. Wissenschaftliche Verlagsgesellschaft $\mathrm{mbH}$ 